\title{
Initial Otoacoustic Emission Hearing Screening Results in Newborns with Patent Ear Canals, Vernix Caseosa and Collapsed Ear Canals
}

\author{
Maria Rina T. Reyes-Quintos ${ }^{1,2,3}$ and Ruby P. Robles ${ }^{3}$ \\ 1Philippine National Ear Institute, National Institutes of Health, University of the Philippines Manila \\ ${ }^{2}$ Department of Otorhinolaryngology, College of Medicine and Philippine General Hospital, University of the Philippines Manila \\ ${ }^{3}$ Department of Otorhinolaryngology Head and Neck Surgery, The Medical City
}

\begin{abstract}
Objectives. This study determined the initial otoacoustic (OAE) hearing screening results of newborns with collapsed ear canals and vernix caseosa in the ear canal and compared these to ears that were patent.

Methods. Two hundred term newborns (400 ears) with normal APGAR scores, birth weight, maternal and gestational history, who were born between August 2013 to October 2013 and who had OAE hearing screening test done by trained midwives were included in this study. All of them underwent otoscopy after the OAE hearing screening test was done to determine patency of the ear canal and presence of vernix caseosa. The examining physician was blinded to the OAE results. Comparison between the $O A E$ results and the otoscopic findings were done.
\end{abstract}

Results. Four hundred ears were included in the study. Two hundred and fifty one ears (62.8\%) had vernix caseosa and 42 ears $(10.5 \%)$ had collapsed ear canal. The overall initial OAE hearing screening test pass rate of the newborns tested was $69.5 \%$. The initial OAE hearing screening test pass rate of newborns those with ear canal vernix caseosa or collapsed ear canal, were $72.1 \%$ and $47.6 \%$, respectively. Patent ears were found in $107(26.7 \%)$ with a pass rate of $71.9 \%$.

Conclusion. The pass rates of ears with vernix caseosa and collapsed ear canal were $72.1 \%$ and $47.6 \%$, respectively. There was no significant difference between the OAE hearing screening test pass rates of ears with patent canal and ears that were collapsed and/ or had vernix caseosa. However, there was a statistically significant difference in pass rates between patent ear canals and collapsed ear canals - with the patent ear canal more likely to pass the OAE hearing screening test than collapsed canals.

Key Words: otoscopy, otoacoustic emissions, newborns, collapsed ear canal, vernix caseosa

Corresponding author: Maria Rina T. Reyes-Quintos, MD, MClinAud Department of Otorhinolaryngology

2nd Floor, Ward 10

Philippine General Hospital

University of the Philippines Manila

Taft Avenue, Ermita, Manila 1000 Philippines

Telephone: +632 5548400 local 2151 or 2152

Email: rinatrq@yahoo.com

\section{Introduction}

Screening the hearing of newborn babies for hearing loss is now the standard of care in many countries around the world. This has led to the early detection of and intervention for hearing loss to minimize the possible negative effects of delayed management; e.g. speech and language delay, social isolation and academic difficulties. The otoacoustic emission (OAE) devices are commonly used for hearing screening because they are portable, quick, easy to use and affordable.

In a tertiary private hospital in Manila, an average of 2,100-2,500 babies are born every year. Ninety six percent of them undergo newborn hearing screening with the OAE newborn hearing screening device. In an unpublished study by Abratique, Batayola and Reyes-Quintos in the same institution, an average of $11.3 \%$ of the newborns fail their initial OAE testing. ${ }^{1}$ An acceptable initial refer rate is $10 \%{ }^{2}$ Some studies have shown that the presence of vernix caseosa and collapsed ear canals lead to initial high refer rates.

The Joint Commission on Infant Hearing (JCIH) in 2007 recommended that all infants should receive hearing screening by 1 month of age, that hearing loss should be identified before three months of age, and that those identified should receive intervention by six months of age. ${ }^{3}$ A number of studies have shown that significantly better language development is associated with early identification of hearing loss, followed by comprehensive intervention before six months of age. ${ }^{4}$ Screening for hearing loss in the newborn period and early intervention has been known to improve the chances that the child diagnosed to have hearing loss will not have lifelong delays in speech and language as well as other problems related to hearing loss. ${ }^{5}$

Devices such as the OAE hearing screening test and the automated auditory brainstem response test (AABR) have been used for hearing screening. OAEs are sounds produced by the outer haircells in response to acoustic signals. These biological sounds are natural by-products of energetic biological processes and their existence provides us with a valuable information on the process of hearing, allowing us to detect the first signs of hearing impairment even in newborn babies. ${ }^{6}$ This test is done by placing a probe in the baby's ear that emits acoustic signals that in turn stimulates the outer hair cells in the cochlea. If hair cells in the inner ear 
are present, it will produce sounds that are then picked up by the OAE microphone.

Many factors have been described to affect the acoustic pattern in the external ear during sound transmission. One of the hypothesized explanations for newborns who fail their initial OAE is due to the presence of vernix caseosa on their external auditory canal or a collapsed canal. ${ }^{7}$

Vernix caseosa, also known as vernix, is the waxy or cheese-like white substance found coating the skin of newborn human babies. While a collapsed ear canal is defined as the inability to visualize the tympanic membrane even with proper maneuvering because the walls of the ear canal are soft and caved in.

Otoscopy is a way of examining the external auditory canal and the tympanic membrane. The examination is performed by gently pulling the outer part of the ear downwards in order to straighten the external auditory canal and inserting a device that illuminates and magnifies the area. This may be more difficult to do in newborns because of their small and compliant ears. Many newborn hearing screening protocols do not involve or emphasize the use of otoscopy prior to initial newborn hearing screening. The individuals performing the screening test may be volunteers, midwives and nurses who may not have the expertise of using an otoscope.

If vernix caseosa and collapsed ear canals greatly influence the result of the newborn OAE hearing screening test, then an otoscopic examination by trained personnel prior to hearing screening should be emphasized as part of the newborn hearing screening protocol. Thus, deferring the OAE hearing screening test for some days until the vernix casoesa has dried or the ear canal has sufficiently stiffened (for those with collapsed ear canals) might then be done.

\section{Materials and Methods}

This is a prospective, cross-sectional study. Included in the study were all term newborns with APGAR score of at least 9,9, with normal maternal history, from the nursery of a private tertiary hospital, born from August 2013 to October 2013. Routine OAE hearing screening test was performed within 24 hours after which otoscopic examination to visualize the patency of their ear canal was done. Results were then recorded and tabulated. No urgent intervention was needed for those with vernix caseosa or collapsing ear canals. Excluded from the study were those with microtia, known ear infection by history and physical examination, congenital or syndromic defects, and a family history of hearing loss.

The otoscopic examination was performed using a Welch Allyn otoscope with a size 2 speculum by a single observer "blinded" to the OAE hearing screening test results. The results were recorded as being patent, with vernix caseosa (complete or partial) or collapsed ear canal. Patent ear canal was defined as the ability to view the entire tympanic membrane (Figure 1). Partial vernix caseosa was the ability to view some parts but not the entire tympanic membrane (Figure 2). And complete vernix caseosa was defined as the inability to view the tympanic membrane at all (Figure 3). A collapsed ear canal was defined as a cavedin canal walls wherein there was inability to visualize the tympanic membrane (Figure 4 ).
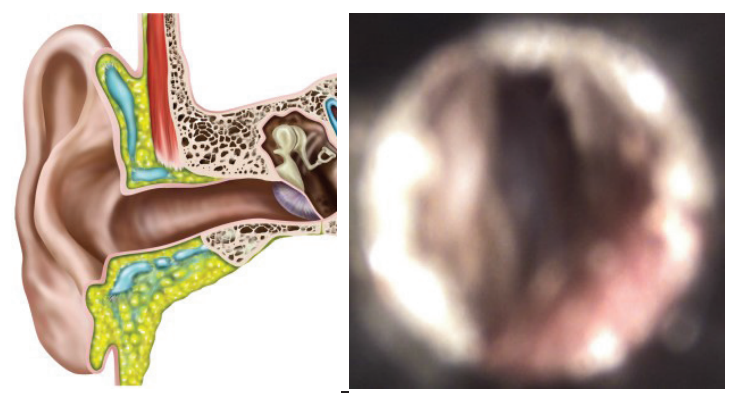

Figure 1. Patent Ear Canal.
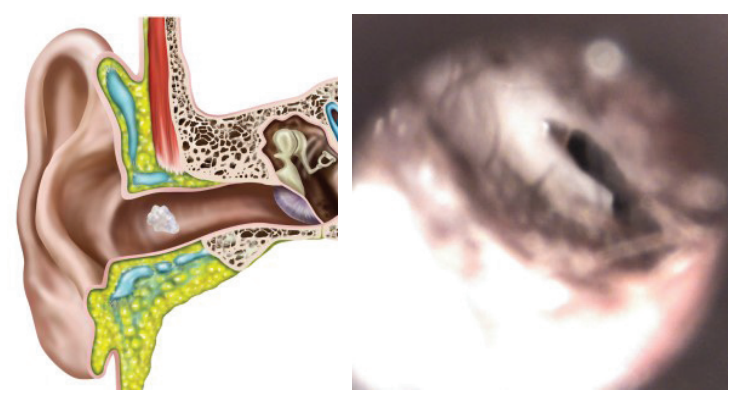

Figure 2. Partial obstruction with vernix caseosa.

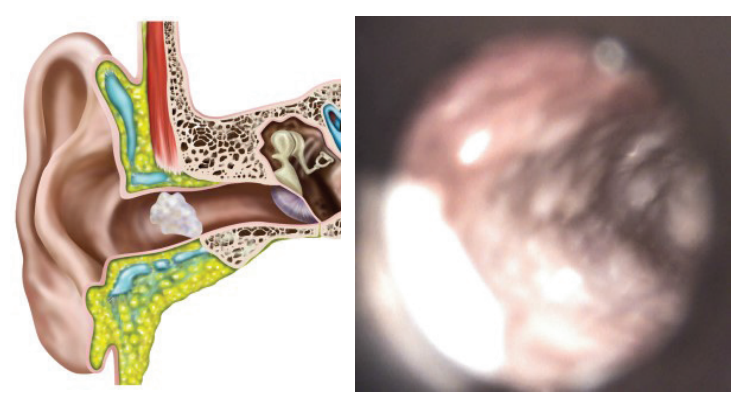

Figure 3. Completely obstructed canal with vernix caseosa.



Figure 4. Collapsed ear canal. 
The OAE testing was done using Otoport Lite by Otodynamics Ltd. by trained midwives in a quiet room at the newborn nursery. All results were recorded as "pass" or "fail" in a logbook. The otoscopic findings were compared to their corresponding OAE hearing screening test result and analyzed statistically. Those who "fail" the screening test are told to return to the Hearing and Dizziness Center for a rescreen after one month.

A Chi-square test and Fisher's exact test were done to determine if there were significant differences between 1) ears that had vernix caseosa and/or collapsed ear canal and those ears that are patent, 2) ears with vernix caseosa and ears that are patent and 3) collapsed ear canals and ear canals that are patent.

This study was approved by the Institutional Review Board (ethical and technical review) of the hospital. All parents of the newborns were informed regarding this study and informed consent was secured. Newborn hearing screening is a standard procedure in the hospital. Universal Newborn Hearing Screening is also mandated by law (RA 9709).

\section{Results}

A total of 200 well newborns comprising of 107 males and 93 females at 2-24 hours of birth were tested accounting for 400 ears. All babies were term at 36-39 weeks and with normal birthweights of 2,540g-3,975g. Patent ears were found in 107 (26.7\%). Vernix caseosa was found in 251 $(62.8 \%)$ ears and collapsed ear canals in 42 (10.5\%). Of the 251 with vernix caseosa in the ear canal, partial vernix caseosa build-up was found in 244 (97.2\%) while 7 had complete vernix caseosa obstructing their canals. Out of 400 ears $278(69.5 \%)$ "passed" the OAE hearing screening test while 122 (30.5\%) ears did not. Out of the 107 patent ears, 77 $(71.9 \%)$ "passed" the OAE hearing screening test. In general, $181(72.1 \%)$ of the ears with vernix caseosa "passed" the OAE screening test. Of the 244 ears with partial vernix caseosa, $174(71.3 \%)$ of them "passed" the OAE hearing screening test and out of the 7 with completely obstructed ears due to vernix caseosa, all "passed" the OAE. Twenty $(47.6 \%)$ of the collapsed ears "passed" the OAE hearing screening test (Table 1).

There was no significant difference between ears that were patent and ears that had vernix caseosa and/or collapsed ear canals (Table 2). When the ear canals with vernix caseosa was analyzed separately from collapsed ear canals and compared to patent ear canals, there was no statistical significant difference in pass rates between ears with patent ear canals and ear canals with vernix caseosa but there was a statistically significant difference in pass rates between ears with patent ear canals and collapsed ear canal (Table 3). A patent ear canal would more likely to pass than collapsed ear canals (Table 4). Only 20 (16.4\%) of the newborns returned for re-screen.
Table 1. Ear canal condition and initial OAE hearing screening results

\begin{tabular}{lrrrrrr}
\hline \multicolumn{1}{c}{ EAR CANAL } & \multicolumn{3}{c}{ OAE RESULTS } & \multicolumn{2}{c}{ TOTAL } \\
\cline { 2 - 7 } \multicolumn{1}{c}{ CONDITION } & Pass & \multicolumn{1}{c}{$\%$} & Fail & \multicolumn{1}{c}{$\%$} & No. & \multicolumn{1}{c}{$\%$} \\
\hline Patent & 77 & $19.2 \%$ & 30 & $7.5 \%$ & $\mathbf{1 0 7}$ & $\mathbf{2 6 . 7 \%}$ \\
Partial vernix & 174 & $43.5 \%$ & 70 & $17.5 \%$ & $\mathbf{2 4 4}$ & $\mathbf{6 1 . 0 \%}$ \\
Complete vernix & 7 & $1.8 \%$ & 0 & $0.0 \%$ & 7 & $\mathbf{1 . 8 \%}$ \\
Collapsed & 20 & $5.0 \%$ & 22 & $5.5 \%$ & $\mathbf{4 2}$ & $\mathbf{1 0 . 5 \%}$ \\
\hline TOTAL & $\mathbf{2 7 8}$ & $\mathbf{6 9 . 5 \%}$ & $\mathbf{1 2 2}$ & $\mathbf{3 0 . 5} \%$ & $\mathbf{4 0 0}$ & $\mathbf{1 0 0 . 0} \%$ \\
\hline
\end{tabular}

Table 2. Chi-Square Test and Fisher's Test Computations of OAE results of patent ear canals and ear canals with vernix caseosa and/or were collapsed

\begin{tabular}{|c|c|c|c|}
\hline \multirow{2}{*}{ EAR CANAL CONDITION } & \multicolumn{3}{|c|}{ OAE RESULTS } \\
\hline & PASS & FAIL & TOTAL \\
\hline PATENT & 77 & 30 & 107 \\
\hline \multicolumn{4}{|c|}{ WITH VERNIX CASEOSA and/or } \\
\hline COLLAPSED & 201 & 92 & 293 \\
\hline TOTAL & 278 & 122 & 400 \\
\hline CHI-SQUARE TEST & \multicolumn{3}{|c|}{ Not significant at $P=0.52$} \\
\hline FISHER'S EXACT TEST & \multicolumn{3}{|c|}{ Not significant at $P=0.54$} \\
\hline
\end{tabular}

Table 3. Chi-Square Test and Fisher's Test Computations of OAE results of patent ear canals and ear canals with vernix caseosa

\begin{tabular}{lrrr}
\hline \multirow{2}{*}{ EAR CANAL CONDITION } & \multicolumn{3}{c}{ OAE RESULTS } \\
\cline { 2 - 4 } & PASS & \multicolumn{1}{c}{ FAIL } & TOTAL \\
\hline PATENT & 77 & 30 & $\mathbf{1 0 7}$ \\
WITH VERNIX CASEOSA & 181 & 70 & 251 \\
TOTAL & 258 & 100 & 358 \\
\hline CHI-SQUARE TEST & Not significant at $P=0.98$ \\
FISHER'S EXACT TEST & Not significant at $P=1.00$ \\
\hline
\end{tabular}

Table 4. Chi-Square Test and Fisher's Test Computations of OAE results of patent ear canals and collapsed ear canals

\begin{tabular}{|c|c|c|c|}
\hline \multirow{2}{*}{ EAR CANAL CONDITION } & \multicolumn{3}{|c|}{ OAE RESULTS } \\
\hline & PASS & FAIL & TOTAL \\
\hline PATENT & 77 & 30 & 107 \\
\hline COLLAPSED & 20 & 22 & 42 \\
\hline TOTAL & 97 & 52 & 149 \\
\hline CHI-SQUARE TEST & \multicolumn{3}{|c|}{ Not significant at $P=0.005$} \\
\hline FISHER'S EXACT TEST & \multicolumn{3}{|c|}{ Not significant at $P=0.007$} \\
\hline
\end{tabular}

\section{Discussion}

The presence of cerumen or vernix in the canal, middle ear effusion and other causes of transient conduction hearing loss have been shown by several studies to interfere with OAE hearing screening test procedures and incur false positive results. Collapsed ear canals, however, have not been studied as extensively. Ear canal debris and middle ear effusion are commonly found in newborn ears especially during the first few days after birth, which may produce mild, temporary conductive hearing loss and result in a "fail" result in the screening program. ${ }^{8}$

Studies have shown that vernix caseosa and collapsed ear canals are factors that can lead to a "fail" result when the child is tested $<48$ hours after birth especially when using the OAE device. ${ }^{9}$ Some institutions have thus kept their fail 
rates low by delaying the performance of their screening test. Furthermore, cleaning the ear canal has increased the pass rates of OAE hearing screening test from $79.0 \%$ to $84.0 \%$ and $76.0 \%$ to $91.0 \%{ }^{4,10}$ In our study, the newborns were tested within 24 hours and the overall initial pass rate was $69.5 \%$. This is comparable to the $70.0 \%$ initial pass rate of newborns tested soon after birth in a study by Olsha $\mathrm{M}$, Newmark M, Bresloff I, et al. ${ }^{11}$ This low initial pass rate and concomitantly high fail rate may also be due to the fact that the babies in the study were tested and examined by otoscopy only several hours apart and that those who did not "pass" have a chance to be tested again the next day if they are not yet cleared for discharge from the hospital.

However, developing countries are known for discharging newborns from the hospital $\leq 24$ hours. ${ }^{9}$ This is because of the increased cost of staying in a hospital, the lack of adequate insurance coverage and need for bed space. It may be difficult to delay discharging these newborns in order to be able to perform newborn hearing screening at a later date.

Compared to other studies, our results show that there are no statistically significant differences in the pass rates among newborns with vernix caseosa alone and those with patent ear canals. Thus the high fail initial rate may be due to other factors other than vernix caseosa. Besides collapsed ear canals, transient middle ear effusion and generally smaller ear canals of Asian newborns may add to the initial fail rate. A study by Couto and Varvallo also did not observe a statistically significant association between the otoacoustic emission screening test results and whether or not the external ear was occluded. ${ }^{12}$

Currently, routine otoscopy on newborns is not performed prior to their OAE hearing screening test despite findings that diagnosis and removal of vernix caseosa increases the pass rates and thus removing the need to return for rescreening within a month after the "fail" screen. This may be because performing otoscopy in all newborns would be personnel intensive and time consuming. Otoscopy is usually reserved for those who return for a rescreen.

The pass rates for those with vernix caseosa and those with collapsed ear canals were $72.1 \%$ and $47.6 \%$, respectively. Surprisingly, those with completely occluded ear canals due to vernix caseosa had a $100 \%$ pass rate. This may be because it is difficult to predict the amount, thickness and density of vernix caseosa present in the ear canal before it affects the transmission of sound from the OAE probe tip. On the other hand, the collapsed ear canal, since it is somehow malleable, may open during manipulation of the OAE probe tip, allowing an opening to be created for the sounds from the screening device to reach and penetrate through the previously closed off ear canal and thus show an otoacoustic emission and "pass" during the test. Therefore, adding otoscopy and removal of vernix caseosa prior to newborn hearing screening may be useful but not practical. Delaying the initial testing to $\geq 48$ hours has been proven to increase the pass rates and is a practical step that our institution has already instituted in order to improve initial pass rates.

The low follow-up rate for rescreen is a very important matter to discuss. To encourage returning for rescreen, the newborns were already scheduled for rescreening prior to discharge from the hospital and their pediatricians routinely inform their patients once they follow-up in a month. The reason for the failure to come for a rescreen needs to be determined so that steps may be taken to address this problem. An unpublished study done in another private hospital showed that the reason cited by $37.4 \%$ of parents who did not bring their baby back for rescreen was because the baby was perceived by the parents to have normal hearing. Although the nurses were taught the proper way to inform the parents of the results, the way the message was imparted and how the parents may have wrongly perceived the message is possible. This has to be investigated.

\section{Conclusion}

This study shows that there is a $69.5 \%$ overall OAE hearing screening test pass rates on newborns in our institution. Collectively, the pass rates for ear canals that were collapsed and/or had vernix caseosa was $68.6 \%$. Separately, the pass rates for those with vernix caseosa and those with collapsed ear canals were $72.1 \%$ and $47.6 \%$, respectively. There was no statistically significant difference in pass rates between patent ear canals and ear canals with that are collapsed and/or had vernix caseosa. There was no statistical significant difference in pass rates between patent ear canal and ears with vernix caseosa. However, there is statistically significant difference in pass rates between patent ear canals and collapsed ear canals - with patent ear canals more likely to pass newborn hearing screening than collapsed ear canals. Clinically, it may not be expedient to perform otoscopy on all newborns prior to newborn hearing screening. This step may be time-consuming and personnel intensive given that only $10.5 \%$ of newborns in this study have collapsed ear canals wherein about half (47.6\%) passed the hearing screening test. Additionally, they may subsequently pass their repeat hearing screening on follow-up after about a month which may give time for their ears to open up. Thus, this means that a preliminary otoscopic examination may not be necessary before initial OAE screening.

\section{References}

1. Abratique RCJ, Batayola MNLC, Reyes-Quintos MRT. Current state of the universal newborn hearing screening program at The Medical City. 2011. Unpublished.

2. Hall JW. Clinical applications of otoacoustic emissions in children. In: Handbook of otoacoustic emissions. California: Singular Publishing Group; 2000. pp. 389-480.

3. American Academy of Pediatrics, Joint Committee on Infant Hearing. Year 2007 position statement: Principles and guidelines for early hearing detection and intervention programs. Pediatrics. 2007; 120(4):898-921. 
4. Doyle KJ, Burggraaff B, Fujikawa S, Kim J, MacArthur CJ. Neonatal hearing screening with otoscopy, auditory brain response and otoacoustic emissions. Otolaryngol Head Neck Surg. 1997; 116(6 Pt 1):597-603.

5. Apuzzo M, Yoshinaga-Itano C. Early identification of infants with significant hearing loss and the Minnesota Child Development Inventory. Semin Hear. 1995;16:124-37.

6. Kemp DT. The OAE story, an illustrated history of OAE research and applications through the first 25 years. Otodynamics Ltd. 2003.

7. Vohr BR, White KR, Maxon AB. Effects of exam procedures on transient evoked otoacoustic emissions (TEOAEs) in neonates. J Am Acad Audiol. 1996; 7(2):77-82

8. Sininger YS, Doyle KJ, Moore JK. The case for early identification of hearing loss in children. Auditory system development, experimental auditory deprivation, and development of speech perception and hearing. Pediatr Clin North Am. 1999;46(1):1-14.
9. van Dyk M, Swanepoel de W, Hall JW $3^{\text {rd }}$. Outcomes with OAE and AABR screening in the first 48 hours. Implications for newborn hearing screening in developing countries. Int J Pediatr Otorhinolaryngol. 2015; 79(7):1034-40.

10. Chang KW, Vohr BR. Norton SJ, Lekas MD. External and middle ear status related to evoked otoacoustic emission in neonates. Arch Otolaryngol Head Neck Surg. 1993; 119(3):276-82.

11. Olsha M, Newmark M, Bresloff I, Feinmesser R, Attias J. Otological evaluation of newborns who failed otoacoustic emission screening. J Basic Clin Physiol Pharmacol. 1999; 10(3):191-200.

12. Couto CM, Carvallo RM. The effect external and middle ears have in otoacoustic emissions. Braz J Otorhinolaryngol. 2009; 75(1):15-23.

13. Sorita RZ. Causes of attrition on repeat OAE testing following the UNHS protocol at St. Luke's Medical Center. 2005. Unpublished.

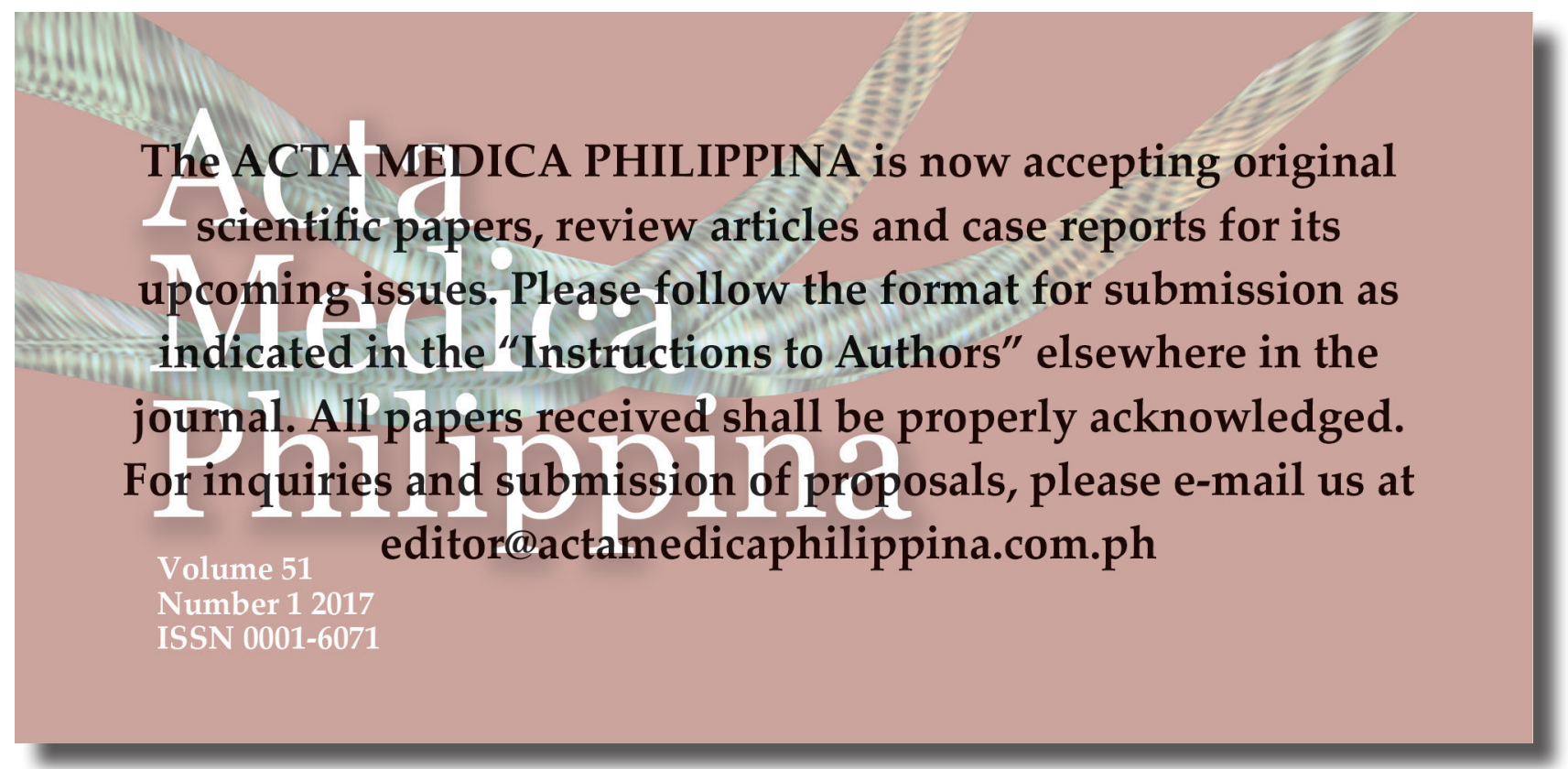

